\title{
Neugeborenenscreening auf Mukoviszidose in Deutschland
}

\section{Cystic Fibrosis Screening in Germany}

\author{
Korrespondenzadresse \\ Ludwig Gortner, Herder-Professor \\ Klinik für Kinder- und Jugendheilkunde \\ Medizinische Universität Wien \\ Währinger Gürtel 18-20 \\ 1090 WIEN \\ Österreich \\ ludwig.gortner@meduniwien.ac.at
}

\author{
Bibliografie \\ DOI http://dx.doi.org/10.1055/s-0043-100167 \\ Klin Padiatr 2017; 229: 55-56 \\ (c) Georg Thieme Verlag KG Stuttgart · New York \\ ISSN 0300-8630
}

Es bedurfte einer Diskussion über nahezu eine Dekade, bis der Gemeinsame Bundesausschuss (G-BA) das Screening auf Mukoviszidose (cystische Fibrose; CF) im Neugeborenenalter beschloss. In zahlreichen anderen Ländern der westlichen Welt wurde das Neugeborenenscreening auf CF bereits seit mehr als 2 Dekaden etabliert, weshalb historisch gesehen der Schritt des G-BA als sehr spät im Vergleich zu anderen Ländern zu betrachten ist. Auf europäischer Ebene ist bereits vor rund 10 Jahren ein Konsensusstatement für die Vorgehensweise verabschiedet worden [4], welches klare Regularien für das Neugeborenenscreening bzw. die Konfirmationsdiagnostik einer CF beinhaltet.

Die Heidelberger Gruppe hat federführend gemeinsam mit anderen Wissenschaftlern aus Deutschland begonnen, die Screeninguntersuchung auf CF basierend auf der Messung des immunreaktiven Trypsins (IRT) sowie eines Pankreas-assoziierten Proteins (PAP) ergänzt durch eine DNA-Untersuchung inkludierend die häufigsten CF-Mutationen prospektiv zu untersuchen. Die Vorteile des 2-stufigen IRT-PAP-Protokolls konnten in einer ersten gemeinsamen Studie im Vergleich zum Screening mittels der IRT-Messung und nachgeschalteter DNA-Analytik von populationsspezifischen Mutationen in unserer bundesdeutschen Population wie oben belegt werden [5]. Diese Daten lagen auch bei Beschlussfassung des G-BA vor, jedoch wurde dort eine Messung des IRT über der 99,9. Perzentile als Marker gewählt und die PAP-Messung vor Analytik von CF-spezifischen Mutationen konzeptionell hintangestellt [1]. Diese Beschlussfassung war Grundlage der Fragestellung der vorliegenden Arbeit der Heidelberger Gruppe, die die Problematik aufgriff und das seitens des G-BA gewählte Screeningkonzept evaluierte.

Hierbei ist das seitens des G-BA gewählte Vorgehen beim zweifachen Nachweis einer IRT-Konzentration aus den Trockenblutfilterkarten > 99,0\% bzw. in der zweiten Probe > 99,9\% verglichen worden mit dem oben erwähnten Heidelberger Protokoll. Dagegen hat das letztere Protokoll, welches als post-hoc Analyse ausgewertet wurde und nunmehr als Publikation vorliegt, eine CF-Positivität erst nach dem zweiten und dritten Schritt der Messung des PAP- sowie der DNA-Analytik bewertet. Als bedeutsamste Unterschiede in den beiden skizzenhaft dargelegten Vorgehensweisen (für die ausführliche Darstellung wird auf das Manuskript der Heidelberger Gruppe verwiesen [6]) fanden sich folgende Daten:
Der relevante Unterschied in den Resultaten beider Protokolle besteht darin, dass aus einem Pool von rund 373,000 getesteten Neugeborenen mittels des G-BA-Protokolls primär 280 falsch-positiv getestete Neugeborene (positiver Vorhersagewert entsprechend rund $20 \%$ ) im Vergleich zum Heidelberger-Protokoll mit nur 31 falsch positiv getesteten Kindern (positiver Vorhersagewert hier rund $70 \%$ ) zu berechnen sind. Einerseits ist die Einführung eines generellen Neugeborenenscreenings auf die CF zu begrüßen, da es jenseits jeden Zweifels belegt ist, dass eine positive Prognose Betroffener sich unter anderem auch an dem frühest möglichen Zeitpunkt der Diagnose festmacht.

Andererseits ist die Akzeptanz eines Screenings mit einer deutlich höheren Rate von falsch-positiven Testresultaten eine Tatsache, die auch bei den Mitgliedern des G-BA reflektiert werden sollte: Hierbei ist insbesondere die Verunsicherung der Familien betroffener Neugeborener zu werten [3], die bis zum definitiven Ausschluss einer CF einer erheblichen Belastung ausgesetzt sind. Darüber hinaus sind die für die weitere Folgediagnostik relevanten Schritte mit einem nicht unerheblichen Kostenaufwand verbunden.

Die CF ist bei der Diagnosestellung atypischer Verläufe noch immer eine Herausforderung [2]. Hinsichtlich des weiteren Vorgehens unter dem zuvor zitierten Aspekt als auch in der vorliegenden Ausgabe der Heidelberger Gruppe vorgelegten Resultate bleiben naturgemäß verschiedene Schritte zu diskutieren. Auf der Basis des bisherigen Sachstandes erscheint es wünschenswert, dass die jetzt publizierten Daten der Heidelberger Gruppe weiter prospektiv untersucht werden, um sicherzustellen, dass nach einer Evaluation der jetzigen Screeningstrategie hierbei die dargestellten Verbesserungen eingebracht werden können, welche in der Zwischenzeit weiter substantiiert und optimiert werden sollten. Naturgemäß bietet sich zum gegebenen Zeitpunkt hier das Heidelberger Protokoll an, da wie in der Publikation ausführlich dargelegt, es zu einer deutlichen Reduktion der Neugeborenen mit falsch-positivem CFTestergebnis kommt. Dies hat für die Akzeptanz des Neugeborenenscreenings und gegebenenfalls einer weiter anstehenden Diskussion um dessen Modifikation einen außerordentlich hohen Stellenwert. Der G-BA sollte die vorliegenden Daten schon jetzt aufnehmen und diese einem Vergleich zu der bisher gehandhabten Screeningstrategie einer kritischen Diskussion unterziehen, um 
einerseits die gesellschaftliche Akzeptanz nicht zu gefährden und möglichst kosteneffizient vorzugehen. Für die hierfür vorgelegten Grundlagen gebührt der Heidelberger Gruppe Dank.

Sicher wird insgesamt die gesellschaftliche Akzeptanz von Screeningmaßnahmen allgemein unter anderem an einer möglichst tiefen Rate an falsch-positiv getesteten Neugeborenen bei einer gegen null gehenden Rate falsch negativer Resultate festgemacht werden.

\section{Literatur}

[1] GemeinsamerBundesausschuss, Kinder-Richtlinie. Änderung des Beschlusses zur Neufassung - Screening auf Mukoviszidose (Zystische Fibrose) - Tragende Gründe zum Beschluss „vom“. doi: https:// www.g-ba.de/informationen/beschluesse/2287/ 2015

[2] Bagheri-Behrouzi A, Steiss JO, Zimmer KP et al. Misdiagnosis of cystic fibrosis based on transient pancreatic insufficiency and elevated sweat chloride. Klin Padiatr 2015; 227: 96-97
[3] Dietrich K, Leiss U, Griessmeier B et al. Parental Distress Caused by Child's Cancer Diagnosis. Klin Padiatr 2016; 228: 149-156

[4] Mayell SJ, Munck A, Craig JV et al. A European consensus for the evaluation and management of infants with an equivocal diagnosis following newborn screening for cystic fibrosis. J Cyst Fibros 2009; 8: 71-78

[5] Sommerburg O, Krulisova V, Hammermann J et al. Comparison of different IRT-PAP protocols to screen newborns for cystic fibrosis in three central European populations. J Cyst Fibros 2014; 13: 15-23

[6] Sommerburg O, Stahl M, Hammermann ] et al. [Neugeborenenscreening auf Mukoviszidose in Deutschland: Vergleich des neuen ScreeningProtokolls mit einem Alternativprotokoll]. Klin Padiatr 2017; 229 